\title{
Posterior Reversible Encephalopathy associated with Eclampsia and HELLP Syndrome
}

\author{
CM Aluwihare $^{1 *}$, S Jayasinghe ${ }^{2}$, H Liyanage $^{2}$ \\ Senior Registrar Critical Care Medicine ${ }^{1 *}$, National Hospital of Sri Lanka, Sri Lanka, Consultant \\ Anaesthetist ${ }^{2}$, De Zoysa Maternity Hospital, Colombo, Sri Lanka.
}

\begin{abstract}
Posterior reversible encephalopathy (PRES) is a rare clinical entity which presents with neurological signs and symptoms with characteristic findings on neuroimaging. Eclampsia is a common pre-disposing cause.

We report a case of a 29-year-old lady presenting at 36 weeks of gestation with eclampsia evident by a very high blood pressure, generalized tonic clonic (GTC) seizures, albuminuria and clonus. She remained drowsy with a fluctuating Glasgow coma scale (GCS) postdelivery with features of HELLP syndrome, prompting the need for neuroimaging which confirmed PRES. Early normal vaginal delivery (NVD), with aggressive blood pressure and seizure control resulted in a complete recovery within 72 hours post-partum.

In a pregnant patient with eclamptic seizures, a high degree of suspicion is needed, and neuroimaging should be utilized in order to diagnose PRES. This case highlights the fact that PRES is a reversible entity with favourable outcomes with timely recognition and aggressive management.
\end{abstract}

Keywords: Posterior reversible encephalopathy syndrome (PRES); eclampsia; HELLP syndrome; pregnancy

\section{Case report}

Mrs S a 29-year-old lady in her third pregnancy, presented at 36 weeks of gestation with epigastric pain and high BP of $150 / 100 \mathrm{mmHg}$ despite being on anti-hypertensives. There was no significant past medical history other than pregnancy induced hypertension in her first pregnancy and the present pregnancy. On admission to ward she was conscious and rational with a BP of $140 / 100 \mathrm{mmHg}$ with normal reflexes and absent proteinuria. She did not have any visual impairment or visual symptoms on admission.

She developed witnessed generalized tonic clonic (GTC) seizures in the ward with a BP of $220 / 140 \mathrm{mmHg}$, epigastric pain, albuminuria and clonus. Intravenous magnesium sulphate was

*Correspondence: CM Aluwihare

E mail: chamali.aluwihare@gmail.com

iD https://orcid.org/0000-0002-3686-7058

Received: $21 / 11 / 2017$

Accepted: 11/12/2017

DOI: http://doi.org/10.4038 /slja.v26i1.8295

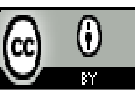

commenced for seizure control. Aggressive BP control was achieved with intravenous hydralazine and a continuous infusion of intravenous labetalol. She was admitted to intensive care unit (ICU) for further management. On admission to ICU, she developed 2 further episodes of seizures. She was drowsy but rousable with a GCS of 12/15 (E2V4M6). Both pupils were normal in size and was equally reactive to light with no focal neurology. Fundoscopy and rest of the neurological examination was normal. There was no fever or neck stiffness. Her lungs were clear. Her urine output was adequate. A decision was made to terminate the pregnancy. However, she was in labour by this time and delivered a healthy baby vaginally.

Patient remained drowsy with a fluctuating low GCS since the first fit. Her BP remained high around $180 / 120 \mathrm{mmHg}$ despite attempts at aggressive BP control, along with albuminuria and clonus. A non-contrast CT (NCCT) of brain showed symmetrical hypodense areas in $\mathrm{B} / \mathrm{L}$ occipital and parietal lobes with diffuse cerebral oedema suggestive of PRES. The MRI of brain showed hypo intense signals on $\mathrm{T}$ - weighted images and hyperintensities in parieto -occipital and temporal lobes on T2-weighted and FLAIR images (Figure 1) confirming the diagnosis of 
PRES. Magnetic resonance arteriogram and the venogram were normal with no evidence of thrombosis. The EEG showed changes suggestive of cortical dysfunction and seizure focus. She was subsequently started on oral levetiracetam by the neurology team. Lupus anticoagulant and anticardiolipin antibodies were not tested since the patient had no history of spontaneous recurrent abortions.

Post-delivery day 1, patient developed HELLP syndrome with elevated liver enzymes (AST546U/L. ALT-189U/L), thrombocytopaenia with a platelet count of $31000 \times 10^{9} / 1$ (previously $159,000 \times 10^{9}$ ) with a blood picture suggestive of microangiopathic haemolytic anaemia. However, the liver functions and the platelet count improved gradually and the LDH levels normalised over the next 24 to 48 hours. She made a full recovery by $72 \mathrm{hrs}$ post-delivery.

Figure 1 MRI brain

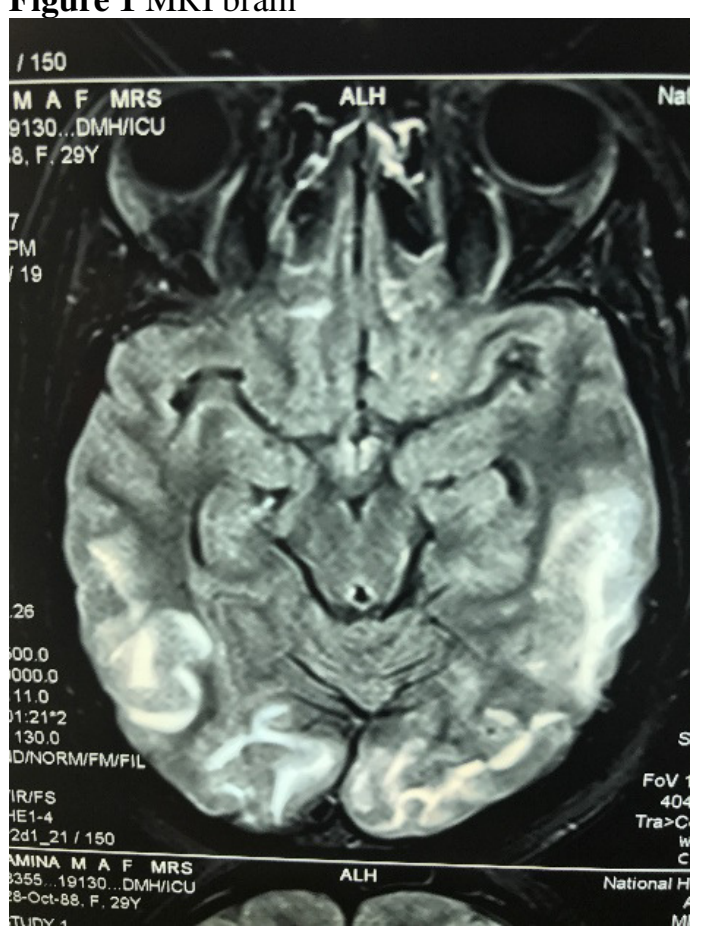

\section{Discussion}

The association of eclampsia and PRES has been shown in many retrospective studies. ${ }^{1,2}$ PRES, previously known as reversible posterior leukoencephalopathy syndrome (RPLS) is a rare clinical and a neuroradiological entity first described by Hinchey et al in $1996 .^{3}$

It is predisposed by many conditions with severe hypertension and systemic inflammatory states.
Examples are malignant hypertension, hypertensive encephalopathy, preeclampsia, eclampsia, renal diseases, cytotoxic and immunosuppressant drugs, blood transfusion, connective tissue disorders and electrolyte disturbances especially low serum magnesium levels ${ }^{3,4}$, infection, sepsis and shock. ${ }^{5}$

The global incidence of PRES is unknown. It has been reported in patients ranging from 4 to 90 years of age. However, most cases have been reported in young to middle-aged adults with a marked female preponderance. ${ }^{3}$

The pathophysiology of PRES is still under debate, but the final common pathway is known to be vasogenic cerebral oedema. This could be caused either by a failure of cerebral autoregulation leading to capillary hyper-perfusion, or endothelial dysfunction leading to vasoconstriction, hypo-perfusion and ischaemia. $^{2}$ This autoregulatory failure is thought to occurs mainly in the posterior circulation. $^{2}$ However, there are multiple postulated mechanisms such as reversible cerebral vasospasm secondary to over reaction of cerebral autoregulation, hyper-perfusion resulting in extravasation of fluid into the brain parenchyma and high intravascular pressures resulting in rupture of the capillaries causing haemorrhage. $^{2}$

PRES is a syndrome characterised by headache, confusion(encephalopathy), seizures and visual loss (less common). ${ }^{3,4}$ However, there is a range of neurological presentations such as nausea, vomiting, headache, confusion, generalised seizures, focal neurological deficit such as cortical blindness, cerebellar syndrome, hemiparesis, finally leading to coma and death. In this context, it is of paramount importance to differentiate from other sinister conditions which can mimic eclampsia or PRES, such as subarachnoid haemorrhage, thrombotic phenomena such as thrombotic thrombocytopaenic purpura, intracranial neoplasm, head trauma, infections such as meningitis an encephalitis, amniotic fluid embolism post-partum angiopathy and idiopathic epilepsy.

There should be a high degree of clinical suspicion whenever there are predisposing causes and should be confirmed by 
neuroimaging modalities. ${ }^{3.4}$ Neuroimaging will show oedema of white matter more in posterior parts of both cerebral hemispheres and parietal occipital white matter in a more symmetrical manner. ${ }^{5,6} \mathrm{MRI}$ is the gold standard for diagnosis of PRES. ${ }^{5,6}$ Retrospective analysis of eclamptic patients have shown a clear association with PRES on imaging, therefore, the question arises as to whether all patients with eclampsia should undergo imaging or should imaging be reserved for those with atypical presentations.

Also, the reversibility of the condition versus the risk of putting a fitting pregnant patient through the scanner may be arguable. We decided on cranial imaging since the patient had a persistent drowsiness and a fluctuating drop in GCS, which we thought was slightly prolonged, in the context of eclampsia despite aggressive BP control. The MRI was confirmatory of PRES with characteristic hypo intense signals in T1weighted and hyperintense signals in parietooccipital regions in T2-weighted and FLAIR images. $^{6}$

The mainstay of treatment of PRES is removal of precipitating cause such as aggressive BP control and seizure control. ${ }^{6}$ Our patient's seizures were controlled with intravenous magnesium sulphate and levetiracetam and the blood pressure was aggressively controlled with intravenous hydralazine and labetalol.

PRES is not always reversible though the name suggests. The outcome of PRES is variable. Prompt diagnosis and timely intervention can lead to complete reversal of clinical and radiological findings with favourable outcomes. However, in a few patients, PRES can progress to ischaemia, infarction with permanent neurological disability and death if the diagnosis or the blood pressure control is delayed. This case highlights the importance of diagnosing PRES in pregnant women presenting with seizures in association with eclampsia and HELLP syndrome although the association is rare.

\section{References}

1. Roth C, Ferbert A. The posterior reversible encephalopathy syndrome: what's certain, what's new? Practical Neurology 2011; 11:136-44. https://doi.org/10.1136/practneurol-2011-000010 PMid:21551107
2. Wagner SJ, Acquah LA, Lindell EP. Posterior Reversible Encephalopathy Syndrome and Eclampsia: Pressing the Case for More Aggressive Blood Pressure Control. Mayo Clin Proc. 2011;86(9):851-856.

https://doi.org/10.4065/mcp.2011.0090 PMid:21878596 PMCid:PMC3258001

3. Hinchey J, Chaves C, Appignani B et al. A reversible posterior leukoencephalopathy syndrome. NEJM 1996; 334:494-500. https://doi.org/10.1056/NEJM199602223340803 PMid:8559202

4. Lindheimer MD, Taler SJ, Cunningham FG. Hypertension in pregnancy. Journal of the American Society of Hypertension 2010;4(2):6878.

https://doi.org/10.1016/j.jash.2010.03.002 PMid:20400051

5. Legriel S, Pico F, Azoulay E. Understanding posterior reversible encephalopathy syndrome. Annual update in intensive care and emergency medicine 2011. Springer, 2011:631-53. https://doi.org/10.1007/978-3-642-18081-1_56

6. Bartynski WS. Posterior reversible encephalopathy syndrome, part 1: Fundamental imaging and clinical features. American Journal of Neuroradiology 2008; 29:1036-42. https://doi.org/10.3174/ajnr.A0928 PMid: 18356474 